\title{
Experiences of international PhD. students under the COVID-19 lockdown: Social closeness in physical distancing times can be an effective stress antidote
}

\author{
Juan Pablo A. Sanchez King, Sanjeev Dahal \\ Boston College, Mexico
}

\begin{abstract}
This exploratory case study is bounded by the COVID-19 lockdown and its impact on six international students pursuing their Ph.D. in the School of Social Work at Boston College. Six online, in depth interviews were conducted with a sample of students from Mexico, Chile, India and Nepal. Data were collected between May and June 2020. Interviews elicited participants narratives on their experiences, including how participants coped under the quarantine, and their views on resources and strategies to reduce stress. Interviews were analyzed, guided by Lazarus and Folkman Transactional Model of Stress and Coping, with a deductive approach to qualitative analysis. Data analysis reveals that the quarantine led to bifurcated consequences. In the positive branch the quarantine provided unexpected conditions allowing students to focus on their work. Additional household and child caregiving roles required that students developed innovative coping practices by adjusting their expectations. On a negative side, students without social networks and community integration experienced greater uncertainties. Both groups experienced strong disrupted emotions, such as fear and anxiety. Findings highlight the importance of social support. For these international $\mathrm{PhD}$. Students, social closeness (in physical distancing times) was an effective stress antidote. Health policies, and programs that aim to prevent and treat stress and burnout among graduate students should focus on the social environment.
\end{abstract}

Keywords: Coping; Resources; Lazarus Theory of Stress; Quarantine. 